\title{
Función renal en cirugía cardíaca con circulación extracorpórea: Pacientes valvulares y coronarios
}

\author{
G uillermo Lema ${ }^{1}$, Roberto Canessa ${ }^{1}$, Jorge U rzúa ${ }^{2}$, \\ Roberto Jalil ${ }^{1}$, Sergio Morán ${ }^{1}$, Claudia Carvajal ${ }^{1}$, \\ Nicolás Aeschlimann ${ }^{1}$, Waldo Merino $^{1}$, María P Jaque ${ }^{1 a}$. \\ Renal function during cardiac surgery \\ with extracorporeal circulation
}

Background: Patients with valvular heart disease are at high risk of acute renal failure after surgery with extracorporeal circulation. Aim: To describe changes in renal function parameters during surgery with extracorporeal circulation in patients with valvular heart disease and compare them with those found in patients undergoing elective coronary surgery. Material and Methods: Two groups of patients were studied. Group 1 was composed by twelve patients undergoing elective coronary surgery and group 2 was composed by eleven patients undergoing surgery for heart valve replacement. Glomerular filtration rate and effective renal plasma flow were estimated from inulin and the ${ }^{131}$ I-hippuran clearance respectively, at five different times, during surgery and the postoperative period. Sodium filtration fraction and fractional excretion were calculated. Alpha and pi-glutathione stransferase in urine were measured as markers of tubular damage in the pre and postoperative periods. Results: Effective renal plasma flow was reduced in both groups before induction of anesthesia, did not change during surgery and decreased significantly in patients with valvular disease in the postoperative period. Glomerular filtration rates were normal during all the study period. There was a non significant reduction of filtration fraction during extracorporeal circulation. Alpha and pi glutathione s-transferases were normal and did not change. Fractional excretion of sodium increased significantly postoperatively. Conclusions: In patients with valvular disease undergoing surgery with extracorporeal circulation, renal function does not deteriorate. No significant difference was found when compared with patients undergoing coronary surgery. No evidence of functional and cellular renal disfunction or damage was found in both study groups (Rev Méd Chile 2008; 136: 459-66).

(Key words: Extracorporeal circulation; Kidney failure; Thoracic surgery)

Recibido el 10 de julio, 2007. Aceptado el 22 de octubre, 2007.

Trabajo financiado por Proyecto FONDECYT \# 1030645-2003.

${ }^{1}$ Departamentos de Anestesiología, Nefrología, y Enfermedades Cardiovasculares, Pontificia Universidad Católica de Chile, Santiago, Chile. ${ }^{2}$ Universidad Santo Tomás, Santiago, Chile.

${ }^{\text {aBioquímica }}$

Correspondencia a: Dr. Guillermo Lema F. Departamento de Anestesiología, Pontificia Universidad Católica de Chile. Marcoleta 367, Santiago, Chile. Fax: 56 (2) 6394409. E mail: glema@med.puc.cl 
$\mathrm{L}$ a disfunción renal es una de las complicaciones más importantes asociada a cirugía cardíaca con circulación extracorpórea (CEC). Tiene un impacto importante en la morbilidad y mortalidad de los pacientes ${ }^{1-5}$. La disfunción renal preoperatoria sigue siendo el predictor independiente más importante de daño renal postoperatorio, en cirugía cardíaca y no cardíaca ${ }^{3}$. Otros predictores son, el uso de inótropos, edad avanzada, insuficiencia cardíaca, enfermedad valvular, cirugía de urgencia y sangrado 3 . Todos ellos, juntos o en forma individual, pueden condicionar disfunción renal o insuficiencia renal aguda. Entre 1\% y 3\% de los pacientes operados con CEC presenta insuficiencia renal aguda, sin embargo un porcentaje muy superior presenta disfunción renal perioperatoria, cuadro de menor mortalidad pero con morbilidad asociada importante.

La CEC ha sido implicada por muchos años en la génesis del daño renal asociado a cirugía cardíaca $^{1-5}$. Diversos estudios de los últimos quince años, usando técnicas sensibles de identificación de daño renal, han sugerido una mayor relevancia del perioperatorio como generador de disfunción e insuficiencia renal aguda, en contraposición al posible daño de la $\mathrm{CEC}^{6}$. La mayor parte de estos estudios ha sido realizada en pacientes coronarios electivos, con función renal preoperatoria normal.

Los pacientes valvulares son considerados un grupo de alto riesgo de daño renal. La mayor parte de los estudios realizados en este grupo de pacientes, es antigua, realizados con técnicas quirúrgicas y de CEC muy disímiles a la tecnología actual $^{7-9}$.

No existen, hasta donde tenemos conocimiento, estudios que evalúen función renal con técnicas sensibles y específicas en pacientes con enfermedad valvular sometidos a cirugía con CEC. Tampoco existen estudios que los comparen con pacientes coronarios usando este tipo de técnicas de estudio.

Estudiamos en forma secuencial y prospectiva, pacientes coronarios y valvulares, sometidos a cirugía con CEC, con función renal preoperatoria normal. Nuestra hipótesis es que la función renal en los pacientes valvulares sometidos a cirugía con CEC, no se compromete significativamente y no muestra diferencias en relación a los cambios que se observan en pacientes coronarios.

\section{MATERIAL Y MÉTODOS}

Este estudio fue realizado previa aprobación de Fondecyt y del Comité de Ética de la Facultad de Medicina de la Pontificia Universidad Católica de Chile. Se obtuvo consentimiento informado, escrito, de todos los pacientes.

Se estudiaron en forma secuencial y prospectiva dos grupos de pacientes sometidos a cirugía cardíaca con CEC: Grupo 1) doce pacientes sometidos a cirugía de revascularización miocárdica electiva, Grupo 2) once pacientes sometidos a cirugía valvular.

Se exigió en todos ellos, creatinina plasmática preoperatoria menor a $1,2 \mathrm{mg} / \mathrm{dl}$.

Se excluyeron los siguientes pacientes: cirugía de urgencia, pacientes inestables hemodinámicamente, uso de inótropos en el perioperatorio, infarto reciente, mayores de 80 años y todos aquellos en los cuales hubo estudios con medio de contraste o uso de drogas nefrotóxicas, al menos $72 \mathrm{~h}$ previo a la cirugía.

Todos los pacientes fueron premedicados con benzodiazepinas y morfina. La anestesia se realizó con fentanyl, midazolam, pancuronium, isoflurano, oxígeno 50\% y aire. La monitorización se realizó mediante electrocardiografía de cinco derivaciones, análisis de segmento ST, presión arterial invasiva, presión venosa central, presión de capilar pulmonar según indicaciones específicas, temperaturas nasofaríngea y rectal. Se monitorizó en forma continua el $\mathrm{O}_{2}$ inspirado y el $\mathrm{CO}_{2}$ de fin de espiración. Se midieron en forma horaria: gases en sangre arterial, tiempo de coagulación activado y glicemia. La orina se recolectó usando una sonda vesical especialmente adaptada para vaciar completamente la vejiga en cada medición ${ }^{10}$.

Luego de la inducción anestésica, se infundieron, $10-15 \mathrm{~mL}^{*} \mathrm{Kg}^{-1}$ de Ringer lactato para mantener presiones de llenado $\pm 20 \%$ del nivel basal. Se monitorizó el débito urinario y se mantuvo sobre $1 \mathrm{~mL}^{*} \mathrm{Kg}^{-1 *} \mathrm{~h}$ previo, y sobre $2 \mathrm{~mL}^{*} \mathrm{Kg}^{-1 * h}$ durante CEC.

La CEC se realizó con bomba de rodillo, flujo no pulsátil, con un oxigenador de membrana, (SX $18^{\circledR}$, Terumo Corp., Tokio, Japón), cebado con 1,51 de Ringer lactato y manitol. Se usó un flujo de 2,22,6 $\mathrm{L}^{*} \mathrm{~min}^{-1 *} \mathrm{~m}^{2}$, hipotermia moderada $\left(28^{\circ} \mathrm{C}\right)$ y el hematocrito mínimo tolerado fue de $23 \%$. Se usó cardioplegia cristaloide St. Thomas I ${ }^{\circledR}$. La presión 
arterial media durante CEC se mantuvo entre $50 \mathrm{y}$ $70 \mathrm{mmHg}$. Se usó fenilefrina y nitroglicerina para tratar los cambios de presión arterial fuera de los valores prefijados.

La técnica anestésica y de manejo de la CEC, fue similar en los dos grupos estudiados.

Estudios de función renal. Se midieron el débito urinario y los electrolitos plasmáticos y urinarios. Se calculó fracción excretada de sodio (FeNa). La filtración glomerular (FG) fue medida mediante clearance de inulina. El flujo plasmático renal (FPRE) fue estimado mediante el clearance de ${ }^{131}$ I-hippuran. Ambas técnicas han sido descritas y validadas previamente ${ }^{10-12}$. Como índice de vasoconstricción o vasodilatación renal se usó la fracción de filtración (FF): filtración glomerular/ flujo plasmático renal. Esta medición ha sido considerada un buen índice del tono de la arteriola eferente ${ }^{13}$.

Todas las mediciones se realizaron, antes de la inducción anestésica, durante CEC en hipo y normotermia, cerrando el esternón y a la hora en el postoperatorio.

El daño tubular renal fue evaluado mediante la medición pre y postoperatoria de alfa y pi glutathione S-Transferase (alfa-GST, pi-GST) en orina (Biotrin NEPHKIT, Biotrin Int. Ltd, Dublin, Ireland). Nuestro grupo tiene experiencia con el análisis de estos marcadores específicos. Otros, quizás más sensibles, tienen costo muy elevado y son de uso excepcional en cirugía cardíaca.

Estadística: Se usaron ANOVA y t de Student. Los datos sin distribución normal, fueron analizados mediante el Wilcoxon signed-rank test. El valor significativo de p se estableció en 0,05. Los datos son expresados como media.

\section{Resultados}

Las características demográficas de ambos grupos se presentan en la Tabla 1. Diez pacientes completaron el estudio en el grupo 1. Dos pacientes en este grupo fueron excluidos por alteraciones hemodinámicas previas a la CEC. Todos los pacientes completaron el estudio en el grupo 2.

No hubo diferencia en los valores de FPRE durante todo el período de estudio (Figura 1). Destacan valores bajos, en ambos grupos de pacientes, al ingreso a pabellón (240 vs $300 \mathrm{~mL} /$ min coronarios vs valvulares, respectivamente), antes de la inducción anestésica, valores que se mantienen disminuidos durante todo el período de estudio. En el grupo de pacientes valvulares existió una disminución significativa de los valores en el postoperatorio, comparado con su valor basal, pero sin diferencia entre los grupos.

La FG fue normal en el pre y postoperatorio (100 y $120 \mathrm{~mL} / \mathrm{min}$, coronarios vs valvulares respectivamente). Existió un descenso no significativo durante cirugía y CEC, sin diferencia entre grupos (Figura 2).

La FF mostró una tendencia a descender durante la CEC, de igual magnitud para ambos grupos, (0,3 y 0,35 coronarios vs valvulares respectivamente), sin diferencia estadísticamente significativa entre grupos y comparado con el basal (Figura 3).

\section{Tabla 1. D atos demográficos para pacientes coronarios (G rupo 1) y valvulares (grupo 2) (promedio y desviación estándar)}

\begin{tabular}{|lcc|}
\hline & Grupo 1 & Grupo 2 \\
\hline № de pacientes & 10 & 11 \\
Hombre & 8 & 5 \\
Mujer & 2 & 6 \\
Edad (años) & $53(34-69)$ & $49(26-77)$ \\
Peso (Kg) & $80,6 \pm 14,2$ & $75,1 \pm 20$ \\
Procedimientos & Cirugía coronaria & Reemplazo valvular: \\
& & Mitral 10 \\
& & Aórtico 1 \\
\hline
\end{tabular}


Rev Méd Chile 2008; 136: 459-466

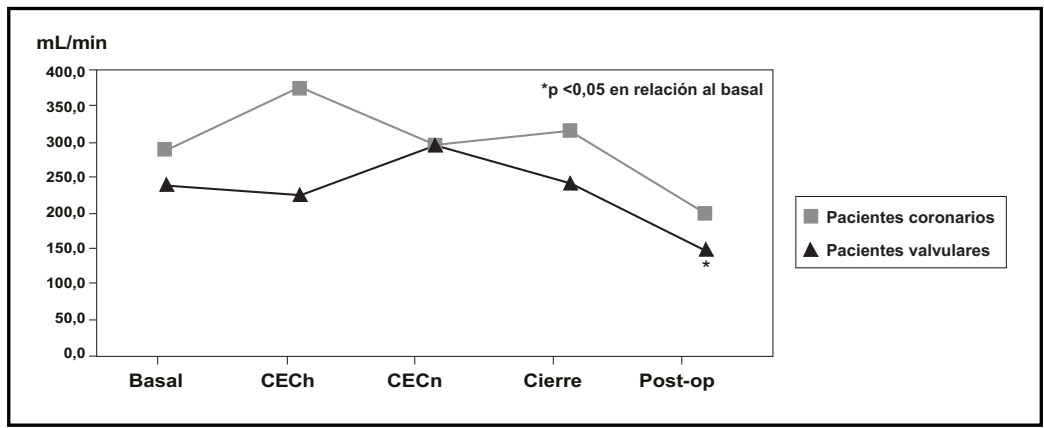

Figura 1. Flujo renal efectivo, pacientes valvulares y coronarios durante cirugía con circulación extracorpórea.

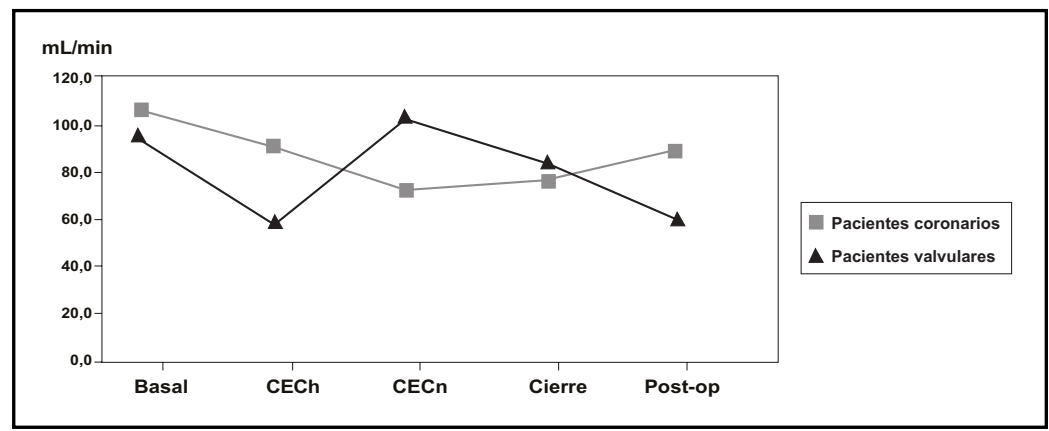

Figura 2. Filtración glomerular, pacientes coronarios y valvulares, durante cirugía con circulación extracorpórea.

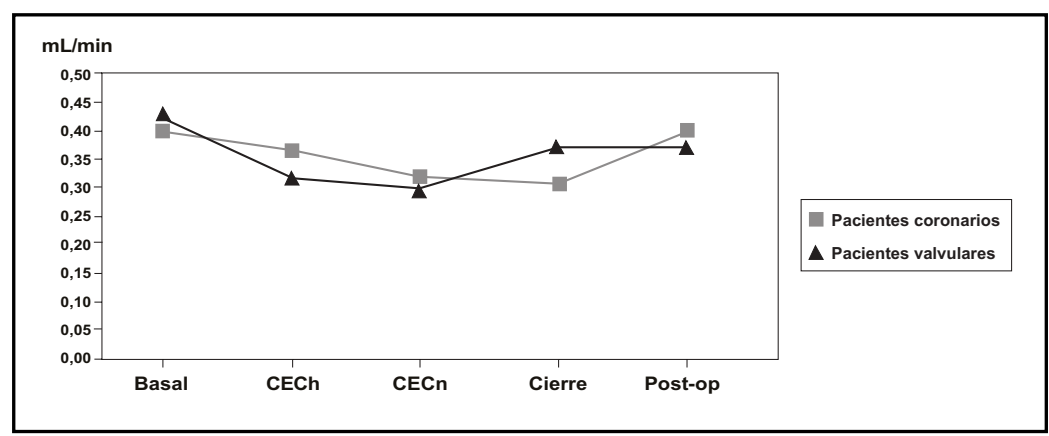

Figura 3. Fracción de filtración, pacientes coronarios y valvulares durante cirugía con circulación extracorpórea.

Los valores de alfa-GST y pi-GST urinaria fueron normales en el pre y postoperatorio, en ambos grupos (Figura 4), incluso al indexarlos por los valores de creatinina urinaria.

La FeNa, normal en el preoperatorio, se elevó significativamente en el postoperatorio, en ambos grupos de pacientes (Figura 5).

\section{DisCUSIÓN}

Este estudio demuestra la ausencia de daño funcional y celular renal en pacientes valvulares y coronarios, con función renal preoperatoria normal, sometidos a cirugía cardíaca con CEC. 


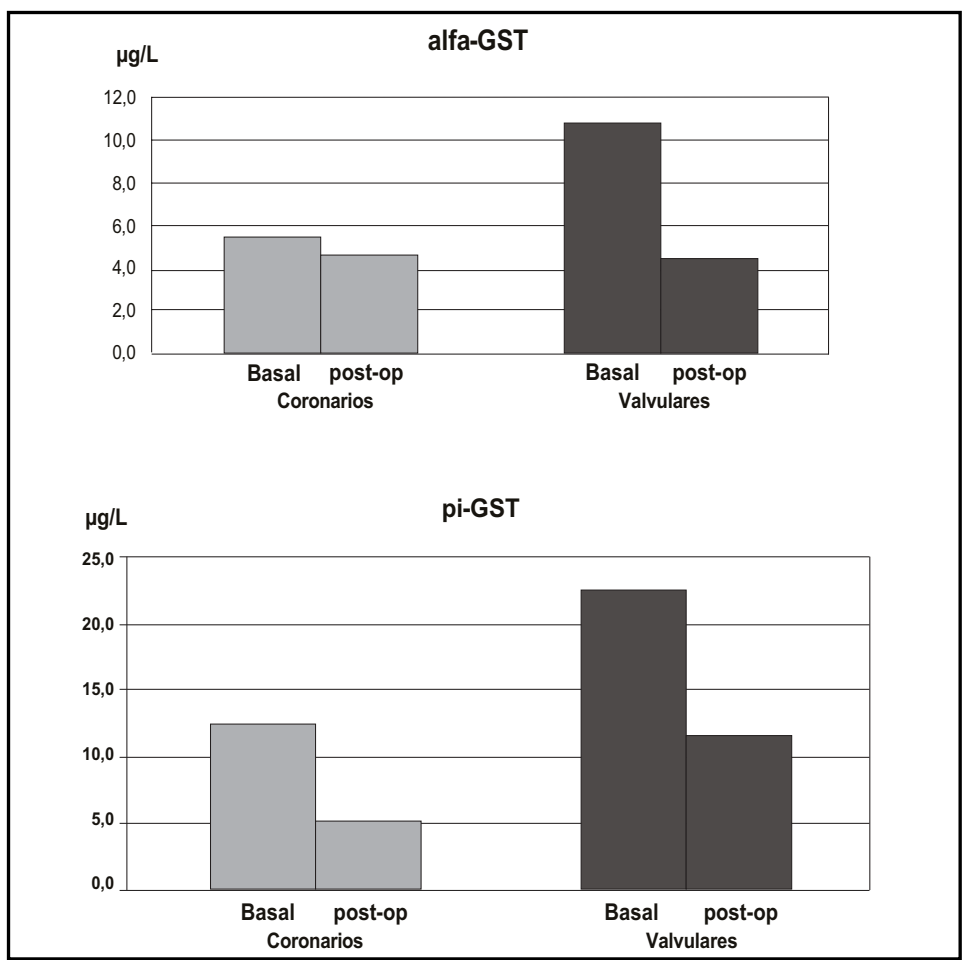

Figura 4. Enzimas Tubulares (alfa-GST y pi-GST), en el pre y post operatorio, en pacientes valvulares y coronarios sometidos a cirugía con circulación extracorpórea.

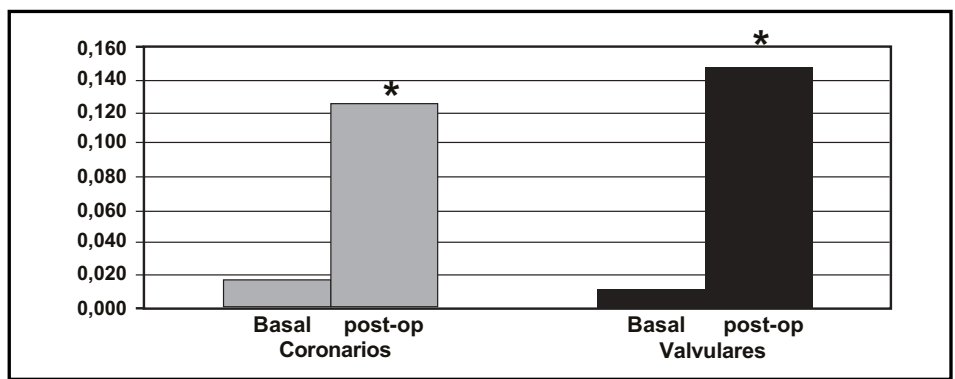

Figura 5. Fracción excretada de sodio, en el pre y post operatorio, pacientes valvulares y coronarios, sometidos a cirugía con circulación extracorpórea.

No existen cambios significativos en los diversos parámetros de función renal estudiados en cada grupo, individualmente con su basal (medición preinducción anestésica), así como cuando ambos grupos son comparados. Existió una reducción significativa en el postoperatorio en los pacientes valvulares.
Durante muchos años se consideró la CEC como el principal y único responsable de disfunción renal e insuficiencia renal aguda en los pacientes cardíacos sometidos a cirugía con $\mathrm{CEC}^{14}$. Durante los últimos veinte años y debido a su prevalencia, la mayor parte de los estudios se concentraron en pacientes coronarios sometidos a 
cirugía cardíaca con CEC. Estos estudios, realizados con técnicas sensibles y específicas para medir función renal, ha permitido aclarar algunos aspectos controvertidos ${ }^{10,11,15,16}$. La CEC en pacientes con función renal preoperatoria normal, no produce daño renal significativo ${ }^{10,15}$. Estudios recientes, comparando cirugía coronaria off pump vs cirugía coronaria convencional, no muestran diferencias en cuanto a daño renal postoperatorio $^{17}$. Estos hallazgos han permitido desmitificar los temores de las técnicas quirúrgicas con CEC. Por otra parte, los estudios han enfatizado el rol del perioperatorio como principal período en el cual se produce este tipo de daños ${ }^{1,6,10,15}$. Hoy está medianamente claro en la literatura, que el principal factor de daño renal postoperatorio es la presencia de disfunción renal preoperatoria: valores sobre 1,5 ó $2,0 \mathrm{mg} / \mathrm{dl}$ de creatinina plasmática parecen ser críticos ${ }^{1-3}$.

Otros factores predisponentes, aunque de menor relevancia son: urgencias quirúrgicas, bajo débito preoperatorio, inestabilidad hemodinámica, uso de inótropos, sangrado postoperatorio, enfermedad valvular y edad avanzada. Todos comparten una particularidad hemodinámica, bajo débito cardíaco y vasoconstricción ${ }^{4}$.

Los pacientes valvulares han sido también considerados dentro del grupo de alto riesgo de presentar daño renal ${ }^{4}$. La mayor parte de los estudios de función renal en pacientes con patología valvular, fueron realizados en la década 196069 y comienzo de 1970-79, previo al aumento de la cirugía coronaria ${ }^{8}$. Estos pacientes, fueron sometidos a procedimientos, con técnicas quirúrgicas, anestésicas, de monitorización y de manejo de la CEC, muy disímiles de las técnicas modernas en uso hoy día. Los estudios de función renal eran simples y al revisarlos retrospectivamente, incompletos. Estos estudios sugerían fuertemente que la CEC era el principal determinante de daño renal postoperatorio, aseveración que se ha repetido en la literatura por años.

El objetivo del presente trabajo fue estudiar los efectos que la CEC produce en la función renal en pacientes con enfermedad valvular, con técnicas muy específicas y sensibles y al mismo tiempo comparar estos resultados con un grupo de pacientes coronarios sometidos también a cirugía. En ambos grupos usamos una técnica estándar de anestesia y manejo de la CEC.
Como métodos de estudio usamos clearance de inulina para el cálculo de la FG. El FPRE fue estimado mediante el clearance de ${ }^{131}$ I-hippuran. Ambas técnicas han sido descritas y usadas previamente por nuestro grupo y otros, y corresponden al gold standard para este tipo de mediciones en el laboratorio y en clínica ${ }^{10,12}$.

Nuestro estudio demuestra que el FPRE está reducido, incluso antes de la cirugía. Este hallazgo ha sido descrito por otros autores y probablemente corresponde a fenómenos de vasoconstricción sistémica y renal asociados a ansiedad, ayuno prolongado, hipovolemia, aumento de los niveles plasmáticos de catecolaminas y otros que probablemente no somos capaces de identificar aún ${ }^{1,16}$. La FeNa reducida en este momento apoya esta hipótesis. El FPRE, se mantiene en los mismos valores del preoperatorio durante el resto del estudio. La CEC no reduce el flujo, sino que lo mantiene en los valores pre bypass. Una reducción significativa del FPRE en el postoperatorio en el grupo 2, pudiese corresponder a episodios de vasoconstricción sistémica y renal, fenómenos conocidos y frecuentes, en pacientes con enfermedad valvular.

La FG es normal en el preoperatorio, se mantiene dentro de límites normales durante la circulación extracorpórea y en el período posterior. Estos hallazgos son opuestos a los descritos para pacientes valvulares, en los cuales siempre se planteó un descenso de la FG durante $\mathrm{CEC}^{7,8,9,14}$.

La FF ha sido señalada como un buen índice de vasodilatación o vasoconstricción de la arteriola eferente. Calculada a partir del FPRE y de la FG, muestra un descenso, aunque no significativo durante CEC, sugiriendo un modesto fenómeno de vasodilatación. Trabajos previos de nuestro grupo demostraron un aumento significativo del FPRE y reducción significativa de la FF durante CEC, sugiriendo vasodilatación ${ }^{10}$. Esta vasodilatación pudo haberse debido a baja viscosidad, hipotermia, o a la liberación de algún péptido vasodilatador durante $\mathrm{CEC}^{18}$. La FF en nuestros dos grupos de estudio, sin embargo, no muestran esta vasodilatación significativa, debido probablemente al uso de técnicas distintas de manejo de la CEC. Hematocritos más altos, menor hipotermia y mayor uso de catecolaminas, para mantener presiones arteriales satisfactorias, pueden ser algunas de las variables que han cambiado. Estas modificaciones han sido sugeridas por estudios en los 
cuáles el outcome parece mejorar, con las modificaciones descritas ${ }^{19-21}$.

Las enzimas alfa-GST y pi-GST son liberadas de los túbulos renales proximales y distales en presencia de necrosis celular ${ }^{22-24}$. Nuestro estudio demuestra que tanto en pacientes coronarios, así como en los valvulares, no existe aumento de estas enzimas en el postoperatorio. Sin embargo, ello sólo refleja necrosis. Pudiese existir daño bioquímico u otras formas de daño celular, que por ahora somos incapaces de evaluar. El estudio de función renal se completó a la hora del postoperatorio, cualquier análisis posterior es extremadamente complejo por la cantidad de variables que pueden influir en el débito cardíaco y la función renal, en este período.

Nuestro estudio muestra aumento significativo de la FeNa, en el postoperatorio. Es probable que

\section{REFERENCIAS}

1. Aronson S, Blumentahl R. Perioperative renal dysfunction and cardiovascular anesthesia: concern and controversies. J Cardiothorac Vasc Anesth 1998; 12: 567-86.

2. St. Andre AC, Del Rossi A. Hemodynamic management of patients in the first hours after cardiac surgery. Crit Care Med 2005; 33: 2082-93.

3. Chertow GM, Burdick E, Honour M, Bonventre JV, BATES DW. Acute kidney injury, mortality, lengh of stay, and costs in hospitalized patients. J Am Soc Nephrol 2005; 16: 3365-70.

4. Grayson AD, Khater M, Jackson M, Fox MA. Valvular heart operation is an independent risk factor for acute renal failure. Ann Thorac Surg 2003; 75: 1829-35.

5. Mangano CM, Diamondstone ls, Ramsay JG, Aggarwal A, Herskowitz A, Mangano DT. Renal dysfunction after myocardial revascularization: risk factors, adverse outcomes and hospital resource utilization. The Multicenter Study of Perioperative Ischemia Research Group. Ann Intern Med 1998; 128: 194-203.

6. Slogoff S, Reul GJ, Keats as, Currv Gr, Crum me, Elmquist BA, Giesecke NM et al. Role of perfusion pressure and flow in mayor organ dysfunction after cardiopulmonary bypass. Ann Thorac Surg 1990; 50: 911-18.

7. Senning A, Andres J, Bornstein $P$, Norberg B, ANDERSEN MN. Renal function during extracorpo- ello refleje un aumento de la carga de sodio, a que son sometidos estos enfermos. La ausencia de alteración de otros parámetros de función renal estudiados apoya esta hipótesis.

\section{Conclusión}

Concluimos que en pacientes valvulares sometidos a cirugía cardíaca con CEC no parece haber evidencia de daño renal, funcional y celular. No existen diferencias al comparar estos resultados con pacientes coronarios sometidos a procedimientos con CEC, ambos grupos con función renal preoperatoria normal. Los hallazgos del presente estudio son consistentes con estudios previos.

real circulation at high and low flow rates: experimental studies in dogs. Ann Surg 1960; 151: 63-70.

8. Porter Ga, Kloster Fe, Herr RJ, Starr A, Griswold HE, Kimsey J. Renal complications associated with valve replacement surgery. J Thorac Cardiovasc Surg 1967; 53: 145-52.

9. Doberneck RC, Reiser MP, LILlehei CW. Acute renal failure after open-heart surgery utilizing extracorporeal circulation and total body perfusion. J Thorac Cardiovasc Surg 1962; 43: 441-52.

10. lema G, Meneses G, Urzúa J, Jalil R, Canessa R, MoRÁN S ET AL. Effects of extracorporeal circulation on renal function in coronary surgical patients. Anesth Analg 1995; 81: 446-51.

11. Lema G, Urzúa J, Jalil R, Canessa R, Morán S, Sacco $\mathrm{C}$ ET AL. Renal protection in patients undergoing cardiopulmonary bypass with preoperative abnormal renal function. Anesth Analg 1998; 86: 3-8

12. lema G, Vogel A, Canessa R, Jalil R, Carvajal C, BECKER P ET AL. Renal function and cardiopulmonary bypass in pediatric cardiac surgical patients. Pediatr Nephrol 2006; 21: 1446-51.

13. Rose BD, Post TW. Renal physiology: proximal tubule. In Rose BD, Post TW. Clinical physiology of acid-base and electrolyte disorders. McGrawHill, fifth edition. 2001; 71-111.

14. Uthey JR, Watchel C, Cain RB, Spaw Ea, Collins JC, STEPHENS DB. Effects of hypothermia, hemodilution and pump oxygenation on organ water 
content, blood flow and oxygen delivery and renal function. Ann Thorac Surg 1981; 31: 121-33.

15. Urzúa J, Troncoso S, Bugedo G, Canessa R, Muñoz H, LEMA G ET AL. Renal function and cardiopulmonary bypass: Effect of perfusion pressure. J Cardiothor Vasc Anesth 1992; 6: 299-303.

16. Colson P, Ribstein J, Mimran A, Grolleau D, Chaptal PA, Roquefeuil B. Effects of angiotensin converting enzyme inhibition on blood pressure and renal function during open-heart surgery. Anesthesiology 1990; 72: 23-7.

17. Chunwuemake A, Weisel A, Maganti M, Nette AF, Wijeysundera DN, Beattie WS, Borger MA. Renal dysfunction in high-risk pataients after on-pump and off-pump coronary artery bypass surgery: a propensity score análisis. Ann Thorac Surg 2005; 80: $2148-53$.

18. Sersar S. Vasomotor dysfunction after cardiac surgery; another look. Eur J Cardiothorac Surg, (Letter). 2005; 27: 529-32.

19. Habib Rh, Zacharias A, Schwann TA, Riordan CJ, Engoren M, Durham SJ, Shah A. Role of hemodilutional anemia and transfusion during cardiopul- monary bypass in renal injury alter coronary revascularization: Implications on operative outcome. Crit Care Med 2005; 33: 1749-56.

20. Dittrich S, Schuth A, Aurich H, vonloeper J, Grosse-Siestrup C, Lange PE. Haemodilution improves organ function during normothermic cardiopulmonary bypass: investigations in isolated perfused pig kidneys. Perfusion 2000; 15: 225-29.

21. Swaminathan M, Philips-Bute BG, Conlon PJ, Smith PK, Newman MF, STAFFORD-Smith M. The association of lowest hematocrit during cardiopulmonary bypass with acute renal injury after coronary artery bypass surgery. Ann Thorac Surg 2003; 76: 784-92

22. Eijkenboom JJ, van Eijk LT, Pickkers P, Peters WHM, Wetzels JFM, van der Hoeven HG. Small increases in the urinary excretion of glutathione S-transferasa A1 and P1 after cardiac surgery are not associated with clinically relevant renal injury. Intensive Care Med 2003; 31: 664-67.

23. Han WK, Bonaventre JV. Biological markers for early detection of acute kidney injury. Curr Opin Crit Care 2004; 10: 476-82. 